\title{
Análisis de ciclo de vida de una nueva solución arquitectónica que mejora el rendimiento térmico de la envolvente del edificio: Fachada Natural Aljibe
}

\section{Life Cycle Assessment of a new architectural solution that improves the thermal performance of the building envelope: Natural Green Tank Façade}

$\underline{\text { R. Carabaño }}^{(*)}$, C. Bedoya ${ }^{(*)}$, D. Ruiz ${ }^{(*)}$

\section{RESUMEN}

El objetivo de este trabajo, que supone la mejora del rendimiento térmico de la envolvente del edificio, es dar a conocer los impactos ambientales asociados a una nueva solución de fachada vegetada mediante la metodología de Análisis de Ciclo de Vida con límites del sistema de la cuna a la tumba. El análisis pretende también generar información relevante de ciclo de vida como material de partida para la redacción de una futura Declaración Ambiental de Producto. La etapa de fabricación de los componentes de la fachada es la que más contribución presenta en todas las categorías de impacto analizadas, siendo la capa vegetada la de mayor impacto. En cambio, la etapa de transporte es la que presenta menor contribución, pudiendo ser considerado despreciable para todas las categorías de impacto analizadas. Tras el análisis de los resultados, se ha obtenido información útil de los aspectos y componentes más problemáticos en el desempeño ambiental de la fachada evaluada, abriendo el camino hacia posibles acciones de reducción de impactos por parte del fabricante.

Palabras clave: Sostenibilidad; impacto ambiental; análisis de ciclo de vida; Declaración Ambiental de Producto (DAP); aislamiento térmico; envolvente del edificio; fachada vegetal aljibe.

\section{ABSTRACT}

The objective of this work, that improves the enclosure's thermal performance of the building, is to divulge the environmental impacts tied to a new solution of vegetated façade by means of the Life Cycle Assessment methodology with a cradle to grave system boundary. The analysis aims also at generating relevant information of the life cycle as base material for drafting a future Environmental Product Declaration. The façade's component manufacture stage is the one presenting the highest contribution for all the analysed impact categories, with the vegetated layer being the one with the greatest impact. Conversely, the transport stage is the one presenting the least contribution, and can be considered negligible for all the analysed impact categories. Following the analysis of the results, we have obtained useful information about the most problematic aspects and components in the assessed façade's performance, thus paving the way towards possible actions of impact reduction by the manufacturer.

Keywords: Sustainability; environmental impact; life cycle assessment; Environmental Product Declaration (EPD); thermal insulation; building enveloping; green tank façade.

(*) Escuela Técnica Superior de Arquitectura - Universidad Politécnica de Madrid (España). Persona de contacto/Corresponding author: rocio.carabano@upm.es (R. Carabaño)

Cómo citar este artículo/Citation: Carabaño, R., Bedoya, C., Ruiz, D. (2014). Análisis de ciclo de vida de una nueva solución arquitectónica que mejora el rendimiento térmico de la envolvente del edificio: Fachada Natural Aljibe. Informes de la Construcción, 66(535): e034, doi: http://dx.doi.org/10.3989/ic.12.128.

Licencia / License: Salvo indicación contraria, todos los contenidos de la edición electrónica de Informes de la Construcción se distribuyen bajo una licencia de uso y distribución Creative Commons Reconocimiento no Comercial 3.o. España (cc-by-nc). 


\section{INTRODUCCIÓN}

Uno de los grandes retos arquitectónicos a día de hoy es la asunción del escenario 450 (1), en el que la Agencia Internacional de la Energía (AIE) ${ }^{1}$ presenta un modelo alternativo para la construcción, tanto a nivel de diseño arquitectónico como urbano, basado en la ecoinnovación y que promueve una mayor eficiencia energética y un menor impacto ambiental.

El proyecto de investigación «SOS Natura, Soluciones Arquitectónicas Vegetales» asume como propio este reto planteado por la AIE y busca dar respuesta a los problemas de eficiencia energética y de impacto ambiental que se plantean en las fachadas de las edificaciones. Su revitalización se fundamenta en el cambio a un modelo más sostenible, basado en sistemas de alta tecnología, que permitan reinventar un elemento de fachada, a nivel de fabricación y puesta en obra, para ofrecer soluciones tanto en el campo de la obra nueva como en el de la rehabilitación.

Uno de los principales objetivos de este proyecto ha sido, por tanto, obtener soluciones arquitectónicas para fachada basadas en el ecodiseño ${ }^{2}$, integrando elementos vegetales para la mejora de la envolvente térmica y su eficiencia energética a lo largo de todo su ciclo de vida.

Como ya ocurriera en la investigación llevada a cabo sobre el sistema Intemper TF de cubierta ecológica aljibe (2), en el que se analizaba el impacto ambiental de los materiales de la cubierta vegetal, en este artículo se analiza también el impacto ambiental de todos los materiales que forman el sistema de Fachada Natural Aljibe.

Para asegurar el cumplimiento de dicho análisis se ha utilizado la metodología del Análisis de Ciclo de Vida (ACV), permitiendo identificar los aspectos a optimizar en el diseño del sistema constructivo.

\section{ACV DE LA FACHADA NATURAL ALJIBE}

El ACV es una metodología para evaluar los aspectos medioambientales y los impactos potenciales asociados a un producto o servicio, mediante la recopilación de un inventario de las entradas y salidas relevantes del sistema bajo estudio, la evaluación de los impactos medioambientales potenciales asociados con esas entradas y salidas y la interpretación de los resultados de las dos fases anteriores, de acuerdo con los objetivos y el alcance establecidos al inicio del estudio. Por entrada y salida se entiende cualquier flujo material o energético que es necesario para que el sistema bajo estudio cumpla las funciones para las que está concebido, así como la generación de residuos, emisiones y efluentes que abandonan dicho sistema (3) (4).
El presente informe, en el que se elabora el ACV de un nuevo sistema de construcción para la envolvente del edificio, sigue las recomendaciones y cumple los requisitos de las normas internacionales ISO 14040:2006 (5) e ISO 14044:2006 (6). De igual modo, se han seguido las recomendaciones de la norma UNE-EN 15804:2012 (7) para que algunos de los resultados del estudio se puedan usar en la elaboración de una Declaración Ambiental de Producto ${ }^{3}$ (DAP). El ACV es una herramienta de información que, por sí sola, tiene poca utilidad para las empresas. Por eso es esencial ligarlo al ecodiseño (mejora) y/o a las DAP (comunicación), dentro de lo que se ha acuñado como Gestión de Ciclo de Vida (8) (9).

\subsection{Objetivos y alcance}

\subsubsection{Objetivo}

El estudio tiene como objetivo el análisis y la evaluación de los impactos ambientales asociados a la fabricación de una solución arquitectónica para la empresa Intemper S.L.

Dicho estudio ha permitido detectar los factores o parámetros con mayor influencia en los impactos ambientales del producto, por lo que la información obtenida podrá ayudar a la empresa a impulsar acciones de mejora ambiental de su producto y a elaborar su propia DAP. En el apartado 2.5 de este artículo se da a conocer tal información.

\subsubsection{Alcance del estudio}

El sistema de cerramiento vegetal, Fachada Natural Aljibe, está formado por paneles compuestos por módulos independientes (Tabla 1) y cumple con los requerimientos higrotérmicos y acústicos del Código Técnico de la Edificación CTE (10), en cuanto a su función como fachada. En la Figura 1 se muestran todos los componentes asociados a la solución propuesta para la envolvente con elementos vegetales y en la Tabla 1 se recoge la descripción de cada uno de los componentes de la misma.

La unidad funcional propuesta es $1 \mathrm{~m}^{2}$ de cerramiento vertical opaco, para edificios con uso terciario, con una duración de 40 años ${ }^{4}$.

Los límites del sistema objeto de este estudio incluyen las siguientes etapas del ciclo de vida de las fachadas:

- Suministro de materias primas y materiales auxiliares

- Transporte hasta lugar de fabricación

- Fabricación

- Transporte hasta obra

- Fin de vida (transporte y proceso de fin de vida)

En cuanto a los procesos, quedan fuera del sistema analizado la producción de maquinaria y equipamiento industrial, de-

\footnotetext{
${ }^{1} \mathrm{http}: / / w w w . i e a . o r g$

2 El ecodiseño considera los impactos ambientales en todas las etapas del proceso de diseño y desarrollo de productos, para lograr productos que generen el mínimo impacto ambiental posible a lo largo de todo su ciclo de vida (UNE 150301: Gestión ambiental del proceso de diseño y desarrollo. Ecodiseño).

${ }^{3}$ Las Declaraciones Ambientales de Producto son documentos que presentan la información ambiental cuantificada sobre el ciclo de vida de un producto, permitiendo la comparación entre productos que cumplan la misma función (UNE-EN ISO 14025: Etiquetas y declaraciones ambientales. Declaraciones ambientales tipo III. Principios y procedimientos).

${ }^{4}$ Esta unidad funcional corresponde al ACV de $1 \mathrm{~m}^{2}$ de cerramiento vertical opaco y para edificios con uso terciario; para adaptar esta información a una DAP, la información relacionada con la duración de vida será la relativa al producto (ver UNE-EN 15804, anexo A).
} 


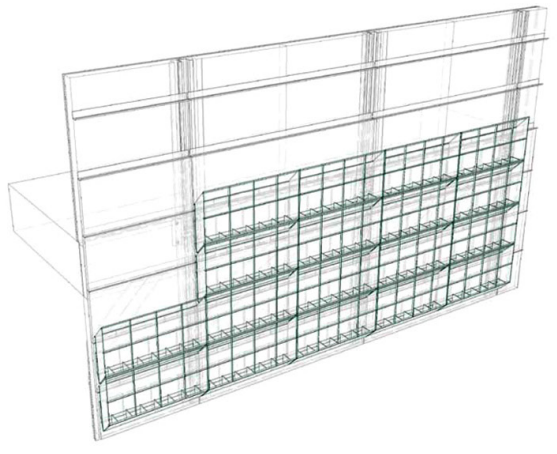

PERSPECTIVA

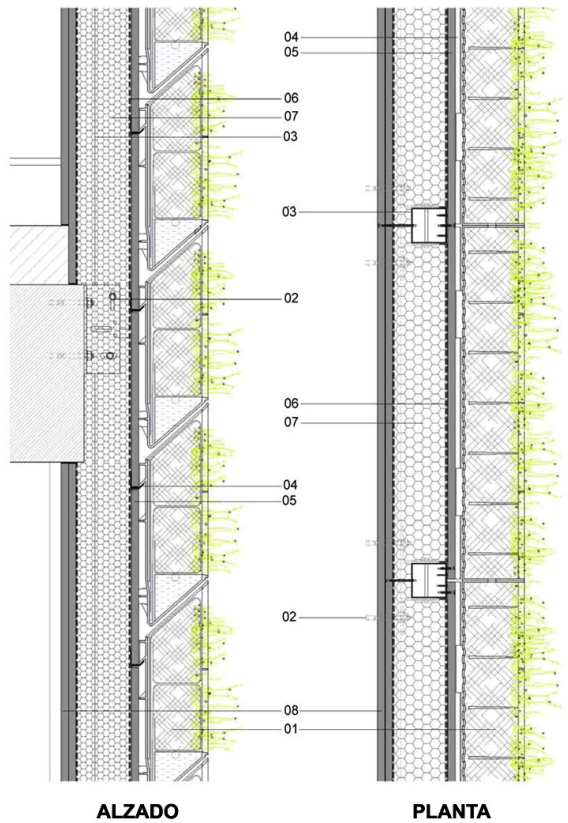

ALZADO

PLANTA

Figura 1. Perspectiva, alzado y planta de la Fachada Natural Aljibe (fuente: equipo de construcción del grupo ABIO-UPM).

bido a la dificultad que supone inventariar todos los bienes implicados. Por otro lado, la comunidad de ACV considera que su impacto ambiental por unidad de producto es bajo, en relación al resto de procesos que sí se incluyen. De esta manera, se han empleado algunas bases de datos en las que no se incluyen dichos procesos. Aquellas bases de datos que sí tienen en cuenta estos procesos han sido adaptadas para cumplir con este criterio. No se ha incluido en el estudio el embalaje de las materias primas ni el embalaje del producto final (Fachada Natural Aljibe), por la dificultad de su modelización, al tratarse de una suma de varios componentes con distintos orígenes hasta su recepción en el lugar de construcción.

En cuanto a las entradas y salidas, se han incluido en el estudio todas aquellas de las que se disponía de información sobre la cantidad y tipo de material, pero se garantiza que aquellas que no se han incluido (por falta de información sobre el material), no suponen más de un $1 \%$ de la suma de materia o energía total inventariada por unidad funcional. La suma de todas las entradas y salidas no inventariadas no excede del $4 \%$ con respecto al valor total, tanto en materia como en energía. El criterio para la exclusión de las entradas y salidas es conforme al apartado 6.3.5. de la norma UNEEN 15804.
En la elaboración del inventario se han utilizado datos proporcionados por Intemper S.L. en lo relativo a composición y cantidad de una parte de los materiales y productos empleados en dicha fachada, así como de las distancias a la que se encuentran los proveedores. Para toda aquella información que no ha sido proporcionada por Intemper S.L., se ha realizado una serie de hipótesis que son expuestas a continuación:

- Materiales y procesos: para inventariar las cargas ambientales asociadas se han utilizado bases de datos comerciales y públicas a través del software GaBi 4.4: European Life Cycle Database (ELCD v.1.o.1), Ecoinvent 2.0 y la desarrollada por PE International.

- Electricidad: a la hora de considerar los impactos ambientales generados en la producción de la electricidad consumida en el sistema bajo estudio, se ha considerado el mix eléctrico español en 2011 calculado a partir de datos suministrados por el Observatorio de la Electricidad del World Wildlife Fund (11).

- Transporte: se han considerado tres tipos de vehículos para el transporte por carretera en territorio nacional, europeo y mundial (ver Tabla 2), según la normativa Euro III (12). En el transporte se diferencian tres etapas: el transporte de las materias primas y productos hasta las insta-

Tabla 1. Descripción de componentes de la Fachada Natural Aljibe.

\begin{tabular}{|l|l|l|}
\hline \multicolumn{2}{|l|}{ CAPA VEGETADA } & 01. Módulos vegetados \\
\hline \multirow{2}{*}{ ESTRUCTURA METÁLICA } & 02. Ménsulas de cuelgue y retención \\
\cline { 3 - 3 } & 03. Subestructura vertical \\
\cline { 3 - 3 } & 04. Subestructura horizontal \\
\hline \multirow{3}{*}{ CAPAS CERRAMIENTO } & Hoja exterior & 05. Paneles de cemento \\
\cline { 2 - 3 } & \multirow{3}{*}{ Hoja intermedia } & 06. Lámina impermeabilizante \\
\cline { 3 - 3 } & & 07. Aislamiento \\
\cline { 2 - 3 } & Hoja interior & 08. Placas de yeso laminado \\
\hline
\end{tabular}


laciones de Intemper S.L.; el transporte de todos los elementos que forman las fachadas propuestas hasta el lugar de construcción del edificio; y el transporte de los restos de demolición hasta vertedero. Para la etapa de transporte hasta proceso de fin de vida se ha supuesto que hay un vertedero de residuos sólidos de construcción en un radio de $80 \mathrm{~km}$ alrededor de la edificación.

- Se supone que todos los componentes de la solución de fachada propuesta tienen una vida útil de referencia que la capacita para cumplir con su función durante el tiempo de vida del edificio, establecido en 40 años, salvo el contenedor NATURPANEL ${ }^{\circledR}$ Aljibe, que se establece en 20 años.

- No se ha incluido en el análisis el uso de agua en servicio de la Fachada Natural Aljibe ya que, por el diseño del contenedor, se entiende que es capaz de recoger y almacenar agua de lluvia. Este consumo es de difícil evaluación, por presentar una gran dependencia de las condiciones climáticas del lugar donde se ubique el edificio (precipitaciones, humedad relativa y temperaturas medias) y de las necesidades hídricas de las especies vegetales implantadas en cada proyecto $\mathrm{y}$, debido a la falta de datos en el contexto del proyecto, ha sido imposible realizar el análisis de escenarios.

- Para los procesos de fin de vida, se ha considerado que los impactos derivados de las operaciones de reciclaje han de ser imputados a los sistemas del producto que los usen como materia prima ${ }^{5}$. Sí se imputan al sistema estudiado todos los procesos relacionados con el depósito en vertedero de los materiales empleados para la fachada, utilizando para ello las bases de datos, tanto de Ecoinvent como de PE International. Los procesos de Ecoinvent relacionados con el fin de vida de los materiales incluyen el transporte hasta vertedero, cosa que no ocurre con los de PE International. Para mantener la coherencia en el uso de las bases de datos se ha optado por adaptar los procesos de Ecoinvent, para que no incluyan dicha fase. Esta etapa del ciclo de vida se ha incluido en el análisis como un proceso por separado.

\subsection{Análisis de inventario}

2.2.1. Suministro de materias primas, transporte, fabricación y transporte hasta la obra

Puesto que la Fachada Natural Aljibe se encuentra dividida en tres sistemas, a continuación se muestran por separado cada uno de ellos:

\subsubsection{Capa vegetada}

El sistema de la capa vegetada se encuentra a su vez dividido en otros tres subsistemas: acabado superficial, contenedor y elementos auxiliares. En la Tabla 2 se muestran los datos de inventario para todos los materiales que componen la capa vegetada, recopilando información relativa a las cantidades del material o componente así como de las etapas de transporte hasta las instalaciones de Intemper S.L. y del transporte de dichos componentes hasta el lugar de construcción del

Tabla 2. Datos de inventario de los componentes de la capa vegetada de la Fachada Natural Aljibe.

\begin{tabular}{|c|c|c|c|c|c|c|c|c|c|c|}
\hline \multirow[b]{2}{*}{ Sistema } & \multirow[b]{2}{*}{ Subsistema } & \multirow[b]{2}{*}{ Elemento } & \multirow[b]{2}{*}{ Material } & \multicolumn{3}{|c|}{$\begin{array}{c}\text { Suministro de materias primas y } \\
\text { materiales auxiliares y Fabricación }\end{array}$} & \multicolumn{2}{|c|}{$\begin{array}{c}\text { Transporte hasta lugar de } \\
\text { fabricación }\end{array}$} & \multicolumn{2}{|c|}{ Transporte hasta obra } \\
\hline & & & & $\begin{array}{l}\text { Material } \\
\text { por } \\
\text { contenedor } \\
\text { (kg) }\end{array}$ & $\begin{array}{l}\text { Material/UF } \\
(\mathrm{kg})\end{array}$ & $\begin{array}{c}\text { Contribución } \\
(\%)\end{array}$ & $\begin{array}{l}\text { Modo de } \\
\text { transporte }\end{array}$ & $\begin{array}{l}\text { Distancia } \\
(\mathbf{k m})\end{array}$ & $\begin{array}{l}\text { Modo de } \\
\text { transporte }\end{array}$ & $\begin{array}{l}\text { Distancia } \\
(\mathrm{km})\end{array}$ \\
\hline \multirow{10}{*}{$\begin{array}{l}\text { Capa } \\
\text { vegetada }\end{array}$} & \multirow{5}{*}{$\begin{array}{l}\text { Acabado } \\
\text { superficial }\end{array}$} & Vegetación & $\begin{array}{l}\text { Según } \\
\text { ubicación del } \\
\text { proyecto }\end{array}$ & $1,50 \mathrm{E}+00$ & 15,37 & 14,26 & \multicolumn{2}{|c|}{ n.a. } & $\begin{array}{l}\text { Camión } \\
\text { 20-26T }\end{array}$ & 200 \\
\hline & & \multirow{3}{*}{ Sustrato vegetal } & $\begin{array}{l}\text { Fibra de coco } \\
(70 \%)\end{array}$ & $1,76 \mathrm{E}+00$ & 18,01 & 16,71 & $\begin{array}{c}\text { Barco - camión } \\
34-40 \mathrm{~T}\end{array}$ & $12000-1000$ & \multirow{4}{*}{$\begin{array}{l}\text { Camión } \\
34-40 \mathrm{~T}\end{array}$} & \multirow{4}{*}{500} \\
\hline & & & $\begin{array}{l}\text { Poliestireno } \\
\text { expandido } \\
\text { granulado, EPS } \\
(30 \%)\end{array}$ & 7,46E-01 & 7,64 & 7,09 & Camión 34-40T & 50 & & \\
\hline & & & $\begin{array}{l}\text { Abono orgánico } \\
\text { NPK («1\%) }\end{array}$ & $2,56 \mathrm{E}-02$ & 0,26 & 0,24 & Camión 34-40T & 1200 & & \\
\hline & & $\begin{array}{l}\text { Fieltro sintético } \\
\text { Feltemper } 150 \\
\text { sustrato }\end{array}$ & $\begin{array}{l}\text { Filamentos } \\
\text { continuos de } \\
\text { poliéster }\end{array}$ & $1,11 \mathrm{E}-01$ & 1,14 & 1,06 & Camión 20-26T & 50 & & \\
\hline & & NATURPANEL & $\begin{array}{l}\text { Polipropileno } \\
\text { (10\% talco) }\end{array}$ & $1,31 \mathrm{E}-02$ & 13,37 & 12,41 & Camión 34-40T & 300 & & \\
\hline & $\begin{array}{l}\text { Sistema } \\
\text { contenedor }\end{array}$ & $\begin{array}{l}\text { Aljibe } \\
\text { (contenedor) }\end{array}$ & $\begin{array}{l}\text { Aditivo } \\
\text { colorante RAL } \\
6006(1,5 \%)\end{array}$ & $1,96 \mathrm{E}-02$ & 0,20 & 0,19 & $\begin{array}{l}\text { Camión hasta } \\
\quad 7 \mathrm{~T}\end{array}$ & 300 & $\begin{array}{l}\text { Camion } \\
34-40 \mathrm{~T}\end{array}$ & 500 \\
\hline & & $\begin{array}{l}\text { Fieltro sintético } \\
\text { Feltemper } 150 \\
\text { auxiliar }\end{array}$ & $\begin{array}{l}\text { Filamentos } \\
\text { continuos de } \\
\text { poliéster }\end{array}$ & 3,10E-02 & 0,32 & 0,29 & Camión 20-26T & 50 & & \\
\hline & $\begin{array}{l}\text { Elementos } \\
\text { auxiliares }\end{array}$ & $\begin{array}{l}\text { Lámina } \\
\text { separadora }\end{array}$ & Policarbonato & $1,80 \mathrm{E}-02$ & 0,18 & 0,17 & $\begin{array}{c}\text { Camión hasta } \\
7 \mathrm{~T}\end{array}$ & 50 & $\begin{array}{l}\text { Camion } \\
34-40 \mathrm{~T}\end{array}$ & 500 \\
\hline & & $\begin{array}{l}\text { Suministro de } \\
\text { agua }\end{array}$ & Caucho & 3,00E-03 & 0,03 & 0,03 & $\begin{array}{c}\text { Camión hasta } \\
7 \mathrm{~T}\end{array}$ & 50 & & \\
\hline
\end{tabular}

\footnotetext{
${ }^{5}$ Este procedimiento es conforme al apartado 6.4.3.3. de la UNE-EN 15804, en el que se establece el límite del sistema del producto de construcción en su etapa de fin de vida cuando el punto de las salidas del sistema en estudio alcanza el estado de fin de residuo.
} 
edificio. Las cantidades de los materiales se expresan en masa por contenedor, masa por unidad funcional y en porcentaje con respecto al peso total de la unidad funcional de la Fachada Natural Aljibe.

Toda la información contenida en dicha tabla corresponde a datos específicos facilitados por Intemper S.L., a excepción del transporte hasta obra, en el que se ha considerado un escenario de distancia media de $500 \mathrm{~km}$ para la península. Los datos relativos a las cantidades de material se han obtenido a partir de mediciones del peso de los componentes, realizados en las instalaciones de Intemper S.L.

\subsubsection{Estructura metálica}

El sistema de la estructura metálica se encuentra a su vez dividido en otros tres subsistemas. En la Tabla 3 se muestran los datos de inventario para todos los materiales que componen la estructura metálica, y donde se recopila información relativa a las cantidades del material o componente, así como de la etapa de transporte hasta el lugar de construcción del edificio. Se entiende que todos estos componentes se transportan desde su lugar de producción/distribución (independiente de Intemper S.L.) hasta la construcción. Para ello se ha considerado un escenario de una distancia media de 100 $\mathrm{km}$, por tratarse de elementos que pueden ser adquiridos de manera local.

Las cantidades de los materiales (tornillos, ménsulas, metros de montante o travesaño, etc.) se expresan en unidades por unidad funcional. Para ninguno de estos componentes se ha contado con datos específicos relativos a su masa. Para su incorporación en el análisis se ha modelizado el volumen de estos componentes, a partir de la información dimensional facilitada por el equipo de construcción del grupo ABIO-UPM. En esta estimación se han usado hipótesis conservadoras, que dan como resultado valores prudentemente superiores a los reales.

Con los valores del volumen calculado y con datos de densidad de los materiales, se ha podido determinar la masa de los componentes por unidad funcional. También se muestra el porcentaje en peso del componente con respecto al peso total de la unidad funcional de la Fachada Natural Aljibe.

\subsubsection{Capas de cerramiento}

El sistema capas de cerramiento se encuentra a su vez dividido en otros tres subsistemas. En la Tabla 4 se muestran los datos de inventario para todos los materiales que componen las capas del cerramiento, y donde se recopila información relativa a las cantidades del material o componente, así como de la etapa de transporte hasta el lugar de construcción del edificio. Se entiende que todos estos componentes se transportan desde su lugar de producción/distribución (independiente de Intemper S.L.) hasta el lugar de construcción del edificio. Para ello se ha considerado un escenario con una distancia de transporte de $100 \mathrm{~km}$, excepto para los paneles de cemento, para los que se ha considerado una distancia de $500 \mathrm{~km}$, al tratarse de un elemento muy específico, con alta probabilidad de no disponer de él de manera local.

Las cantidades de los materiales se expresan en unidades (tornillos, metros de cinta para juntas, $\mathrm{m}^{2}$ de panel, etc.) por unidad funcional. Para ninguno de estos componentes se ha contado con datos específicos relativos a su masa. Para su incorporación en el análisis se ha modelizado el volumen de estos componentes, a partir de la información dimensional facilitada por el equipo de construcción del grupo ABIOUPM. Con los valores del volumen calculado y con valores de la densidad de los materiales, se ha podido determinar la masa de los componentes por unidad funcional. También se muestra el porcentaje en peso del componente, con respecto al peso total de la unidad funcional de la fachada natural aljibe.

\subsubsection{Fin de vida: transporte y proceso de fin de vida}

Para la modelización de los procesos de fin de vida de los materiales relacionados con la fachada se han creado dos grupos. Por un lado, se han agrupado aquellos materiales para los que existe, en la actualidad, un importante mercado relacionado con su reciclaje, y por otro lado, aquellos materiales que tienen una elevada probabilidad de acabar en el vertedero.

En la Tabla 5 se muestran los componentes de la Fachada Natural Aljibe clasificados entre los materiales que son reciclados y aquellos que son llevados a vertedero. Para los com-

Tabla 3. Datos de inventario de los componentes de la estructura metálica de la Fachada Natural Aljibe.

\begin{tabular}{|c|c|c|c|c|c|c|c|c|c|c|}
\hline & & & & Suminist & $\begin{array}{l}\text { de materias prime } \\
\text { lugar de }\end{array}$ & $\begin{array}{l}\text { y material } \\
\text { oricación y } 1\end{array}$ & $\begin{array}{l}\text { auxiliares, } \operatorname{Tr} \\
\text { bricación }\end{array}$ & insporte hasta & Transporte & hasta obra \\
\hline Sistema & Subsistema & Elemento & Material & $\begin{array}{l}\text { Cantidad } \\
\text { UF }\end{array}$ & $\begin{array}{l}\text { Volumen/unidad } \\
\left(\mathrm{cm}^{3}\right)\end{array}$ & $\begin{array}{c}\text { Densidad } \\
\left(\mathrm{g} / \mathrm{cm}^{3}\right)\end{array}$ & $\begin{array}{l}\text { Material/UF } \\
\left(\mathbf{1 ~ m}^{2}\right)(\mathrm{kg})\end{array}$ & $\begin{array}{c}\text { Contribución } \\
\text { (\%) }\end{array}$ & $\begin{array}{l}\text { Medio de } \\
\text { transporte }\end{array}$ & $\begin{array}{c}\text { Distancia } \\
(\mathrm{km})\end{array}$ \\
\hline \multirow{6}{*}{$\begin{array}{l}\text { Estructura } \\
\text { metálica }\end{array}$} & \multirow{2}{*}{$\begin{array}{l}\text { Ménsulas } \\
\text { de cuelgue y } \\
\text { retención }\end{array}$} & Ménsula & $\begin{array}{l}\text { Acero } \\
\text { laminado }\end{array}$ & 1,233 ud & 73,2 & 7,85 & 0,7 & 0,65 & \multirow{6}{*}{$\begin{array}{l}\text { Camión } \\
20-26 \mathrm{~T}\end{array}$} & \multirow{6}{*}{100} \\
\hline & & $\begin{array}{l}\text { Tacos } \\
\text { (fijación al } \\
\text { forjado) }\end{array}$ & $\begin{array}{l}\text { Acero } \\
\text { inoxidable } \\
\text { AISI-304 }\end{array}$ & $2,47 \mathrm{ud}$ & 4,56 & 7,93 & 0,09 & 0,08 & & \\
\hline & \multirow[b]{2}{*}{$\begin{array}{l}\text { Subestructura } \\
\text { vertical }\end{array}$} & Montantes & $\begin{array}{l}\text { Acero } \\
\text { laminado }\end{array}$ & $1,67 \mathrm{~m}$ & 480 & 7,85 & 6,29 & 5,84 & & \\
\hline & & $\begin{array}{l}\text { Pasadores } \\
\text { (fijación } \\
\text { a las } \\
\text { ménsulas) } \\
\end{array}$ & $\begin{array}{l}\text { Acero } \\
\text { inoxidable } \\
\text { AISI-304 }\end{array}$ & 1,233 ud & 1,73 & 7,93 & 0,02 & 0,02 & & \\
\hline & \multirow{2}{*}{$\begin{array}{l}\text { Subestructura } \\
\text { horizontal }\end{array}$} & Travesaños & $\begin{array}{l}\text { Acero } \\
\text { conformado } \\
\text { en frio }\end{array}$ & $3,33 \mathrm{~m}$ & 85,5 & 7,85 & 2,24 & 2,07 & & \\
\hline & & $\begin{array}{l}\text { Tornillos } \\
\text { (fijación al } \\
\text { montante) }\end{array}$ & $\begin{array}{l}\text { Acero } \\
\text { inoxidable } \\
\text { AISI-304 }\end{array}$ & $11,12 \mathrm{ud}$ & 0,17 & 7,93 & 0,01 & 0,01 & & \\
\hline
\end{tabular}


Tabla 4. Datos de inventario de los componentes de las capas de cerramiento de la Fachada Natural Aljibe.

\begin{tabular}{|c|c|c|c|c|c|c|c|c|c|c|}
\hline \multirow[b]{2}{*}{ Sistema } & \multirow[b]{2}{*}{$\begin{array}{l}\text { Subsiste- } \\
\text { ma }\end{array}$} & \multirow[b]{2}{*}{ Elemento } & \multirow[b]{2}{*}{ Material } & \multicolumn{5}{|c|}{ Suministro de materias primas, materiales auxiliares y Fabricación } & \multicolumn{2}{|c|}{ Transporte hasta obra } \\
\hline & & & & Cantidad/UF & $\begin{array}{c}\text { Volumen/unidad } \\
\left(\mathrm{cm}^{3}\right)\end{array}$ & $\begin{array}{c}\text { Densidad } \\
\left(\mathrm{g} / \mathrm{cm}^{3}\right)\end{array}$ & $\begin{array}{l}\text { Material/UF } \\
\left(1 \mathrm{~m}^{2}\right)(\mathrm{kg})\end{array}$ & $\begin{array}{c}\text { Contribución } \\
\text { (\%) }\end{array}$ & $\begin{array}{c}\text { Modo de } \\
\text { transporte }\end{array}$ & $\begin{array}{c}\text { Distancia } \\
(\mathrm{km})\end{array}$ \\
\hline \multirow{7}{*}{$\begin{array}{l}\text { Capa de } \\
\text { cerramiento }\end{array}$} & \multirow{3}{*}{$\begin{array}{l}\text { Hoja } \\
\text { exterior }\end{array}$} & $\begin{array}{l}\text { Paneles de } \\
\text { cerramiento }\end{array}$ & $\begin{array}{l}\text { Cemento, } \\
\text { árido ligero y } \\
\text { fibra de vidrio }\end{array}$ & $1 \mathrm{~m}^{2}$ & - & $\begin{array}{c}15,8 \\
\left(\mathrm{~kg} / \mathrm{m}^{2}\right)\end{array}$ & 15,8 & 14,66 & $\begin{array}{l}\text { Camión } \\
34-40 \mathrm{~T}\end{array}$ & 500 \\
\hline & & $\begin{array}{l}\text { Cinta para } \\
\text { juntas }\end{array}$ & $\begin{array}{l}\text { Espuma } \\
\text { polietileno }\end{array}$ & $3,33 \mathrm{~m}$ & 37,50 & 0,024 & $2,97 \mathrm{E}-03$ & 0 & $\begin{array}{l}\text { Camión } \\
\text { hasta } 7 \mathrm{~T}\end{array}$ & 100 \\
\hline & & $\begin{array}{l}\text { Tornillos de } \\
\text { fijación al } \\
\text { montante }\end{array}$ & $\begin{array}{l}\text { Acero } \\
\text { inoxidable } \\
\text { AISI-304 }\end{array}$ & $6,68 \mathrm{ud}$ & 0,31 & 7,93 & $6,01 \mathrm{E}-03$ & 0,01 & $\begin{array}{l}\text { Camión } \\
\text { hasta } 7 \mathrm{~T}\end{array}$ & 100 \\
\hline & \multirow{2}{*}{$\begin{array}{l}\text { Hoja } \\
\text { intermedia }\end{array}$} & Aislante & Lana de roca & $1 \mathrm{~m}^{2}$ & 90000,00 & 0,04 & 3,6 & 3,34 & $\begin{array}{l}\text { Camión } \\
\text { 34-40T }\end{array}$ & 100 \\
\hline & & $\begin{array}{l}\text { Lámina } \\
\text { impermeable }\end{array}$ & $\begin{array}{l}\text { Polietileno } \\
\text { alta densidad }\end{array}$ & $1 \mathrm{~m}^{2}$ & - & $\begin{array}{c}60 \\
\left(\mathrm{~g} / \mathrm{m}^{2}\right) \\
\end{array}$ & 0,06 & 0,06 & $\begin{array}{l}\text { Camión } \\
\text { 20-26T }\end{array}$ & 100 \\
\hline & \multirow{2}{*}{ Hoja interior } & $\begin{array}{l}\text { Paneles } \\
\text { de yeso } \\
\text { laminado } \\
\end{array}$ & Yeso-cartón & $2 \mathrm{~m}^{2}$ & - & $\begin{array}{c}11,2 \\
\left(\mathrm{~kg} / \mathrm{m}^{2}\right)\end{array}$ & 22,4 & 25,99 & $\begin{array}{l}\text { Camión } \\
34-40 \mathrm{~T}\end{array}$ & 100 \\
\hline & & $\begin{array}{l}\text { Tornillos de } \\
\text { fijación al } \\
\text { montante }\end{array}$ & $\begin{array}{l}\text { Acero } \\
\text { inoxidable } \\
\text { AISI-304 }\end{array}$ & 6,68 ud & 0,36 & 7,93 & $2,03 \mathrm{E}-02$ & 0,02 & $\begin{array}{l}\text { Camión } \\
\text { hasta } 7 \mathrm{~T}\end{array}$ & 100 \\
\hline
\end{tabular}

Tabla 5. Procesos de fin de vida de los componentes de la Fachada Natural Aljibe.

\begin{tabular}{|c|c|c|c|c|c|}
\hline Sistema & Elemento & Material & $\begin{array}{c}\text { Proceso fin } \\
\text { de vida }\end{array}$ & Tratamiento en vertedero & $\begin{array}{c}\text { Cantidad } \\
(\mathrm{kg})\end{array}$ \\
\hline \multirow{6}{*}{ Capa vegetada } & Contenedor & Polipropileno con talco & Reciclaje & - & 13,57 \\
\hline & \multicolumn{2}{|c|}{ Poliestireno expandido } & \multirow{4}{*}{ Vertedero } & \multirow{4}{*}{ Mezcla de plásticos } & \multirow{4}{*}{9,31} \\
\hline & Fieltro & Fibras de poliester & & & \\
\hline & Lámina separadora & Policarbonato & & & \\
\hline & Suministro de agua & Caucho & & & \\
\hline & \multicolumn{2}{|l|}{ Fibra de coco } & Vertedero & Basura orgánica & 18,01 \\
\hline \multirow{2}{*}{$\begin{array}{l}\text { Estructura } \\
\text { metálica }\end{array}$} & Perfiles y ménsulas & \multirow{2}{*}{ Acero } & \multirow{2}{*}{ Reciclaje } & \multirow{2}{*}{-} & \multirow{2}{*}{9,35} \\
\hline & Fijaciones & & & & \\
\hline \multirow{5}{*}{$\begin{array}{l}\text { Capas de } \\
\text { cerramiento }\end{array}$} & Paneles de yeso & & \multirow{2}{*}{ Vertedero } & \multirow{2}{*}{ Material inerte (no especifico) } & \multirow{2}{*}{38,2} \\
\hline & Paneles de cemento & & & & \\
\hline & Fijaciones & Acero inoxidable & Vertedero & Acero & 0,03 \\
\hline & Aislantes & Lana de roca & Vertedero & Lana mineral & 3,6 \\
\hline & Cintas de juntas & Polietileno & Vertedero & Mezcla de plásticos & 0,06 \\
\hline
\end{tabular}

ponentes que son llevados a vertedero, se muestra también el tratamiento que se les ha dado en el modelo de ACV.

En el grupo de los componentes reciclados se encuentran todos los elementos de la estructura metálica ya que, los materiales de que está compuesta tienen un alto valor económico en el mercado de reciclaje. Dentro de este grupo, se ha incluido también el contenedor, ya que es un elemento lo suficientemente voluminoso como para facilitar su separación y asegurar la rentabilidad de su reciclaje. En la actualidad, es una práctica común el reciclaje de plástico; de hecho el polipropileno con el que está fabricado el contenedor tiene este origen.

Dentro del segundo grupo de materiales, los destinados al vertedero, se encuentran aquellos para los que actualmente no hay un tratamiento generalizado de reciclaje o recuperación. Se incluyen también en este grupo los tornillos de fijación de los paneles de yeso, así como todos los materiales plásticos que acompañan al contenedor. Estos elementos se han modelizado con un fin de vida en vertedero, ya que, a pesar de estar fabricados con materiales reciclables, sus dimensiones son pequeñas, afectando a la rentabilidad de las operaciones de reciclaje y a la probabilidad de su separación durante la demolición del edificio.

El transporte de los materiales hasta el vertedero se ha modelizado mediante un camión de 20-26 toneladas clase Euro III, con un recorrido de $80 \mathrm{~km}$.

\subsection{Evaluación de impactos e interpretación}

Las categorías de impacto evaluadas en este trabajo corresponden a las metodologías Ecoindicador 99 (13) y CML 2001 (14), que representan el estado del arte de las metodologías de punto final o endpoint y de punto intermedio o midpoint respectivamente. Para su cálculo se ha empleado el software GaBi 4.4 desarrollado por PE International.

Las categorías de impacto analizadas según la metodología CML 2001 son:

- Potencial de agotamiento de los recursos materiales no renovables o agotamiento de recursos abióticos (AA), expresado en $\mathrm{kg}$ de $\mathrm{Sb}$. 
- Potencial de acidificación de recursos hídricos y del suelo (A), expresado en $\mathrm{kg}$ de $\mathrm{SO}_{2}$ equivalente.

- Potencial de eutrofización (Eu), expresado en $\mathrm{kg}$ de $\mathrm{PO}_{4}^{-3}$ equivalente.

- Potencial de calentamiento global (CG), expresado en kg de $\mathrm{CO}_{2}$ equivalente.

- Potencial de agotamiento del ozono estratosférico (AO), expresado en $\mathrm{kg}$ de $\mathrm{R}_{11}$ equivalente.

- Potencial de formación de ozono fotoquímico (OF), expresado en $\mathrm{kg}$ de etano equivalente.

- Toxicidad humana (TH), expresado en kg de 1,4-diclorobenceno (DCB) equivalente.

- Ecotoxicidad de agua dulce (EAD), expresado en $\mathrm{kg}$ de 1,4-diclorobenceno (DCB) equivalente.

- Ecotoxicidad acuática marina (EAM), expresado en kg de 1,4-diclorobenceno (DCB) equivalente.

Las categorías de impacto analizadas según el Ecoindicador 99 son:

- Salud humana: carcinogénicos (C), orgánicos respirables (OR), inorgánicos respirables (IR), cambio climático (CC), deterioro de la capa de ozono (CO) y radiación ionizante (RI), todas ellas expresadas en Disability Life Years (DALY): años de vida sometidos a una discapacidad (número de años de vida perdidos y el número de años en los que se ha sufrido una enfermedad), terminología empleada por la OMS.

- Calidad del ecosistema: ecotoxicidad (E) y acidificación/ eutrofización (A/E). Expresadas como Potentially Disappeared Fraction (PDF) o fracción de especies que potencialmente desaparecerán (plantas vasculares y organismos sencillos) como consecuencia del impacto ambiental experimentado en un área durante un tiempo determinado $\left(\mathrm{PDF} \cdot \mathrm{m}^{2} \cdot \mathrm{a}\right)$.
- Conservación de recursos: minerales (M) y combustibles fósiles (CF). Expresadas como MJ de energía extra que será necesaria para la futura extracción de mineral de baja calidad y de recursos fósiles.

\subsubsection{Suministro de materias primas, transporte y fabricación}

En las Tablas 6 y 7 se muestra la evaluación de las categorías de impacto asociadas a la etapa de suministro de materias primas, transporte y fabricación, según la metodología CML 2001 y Ecoindicador 99 respectivamente. En esta evaluación ha quedado excluida la fijación del carbono biogénico durante el crecimiento de la fibra de coco, siendo su valor de $94 \mathrm{~kg}$ de $\mathrm{CO}_{2}$, ya que se considera que éste volverá a ser emitido al final del ciclo de vida en forma de $\mathrm{CH}_{4}$. En la Figura 2 se muestra la contribución relativa, en porcentaje y para cada metodología, de cada uno de los sistemas que componen la fachada.

\subsubsection{Transporte hasta obra}

En las Tablas 8 y 9 se muestra la evaluación de las categorías de impacto asociadas a la etapa de transporte de los elementos de la Fachada Natural Aljibe hasta el lugar de construcción del edificio, según la metodología CML 2001 y Ecoindicador 99 respectivamente.

\subsubsection{Fin de vida: transporte y eliminación final}

En las Tablas 10 y 11 se muestra la evaluación de las categorías de impacto asociadas a los procesos de fin de vida de los materiales asociados a la Fachada Natural Aljibe, según las metodologías CML 2001 y Ecoindicador 99, respectivamente. Estos impactos se encuentran desglosados por los conjuntos

Tabla 6. Evaluación de las categorías de impacto (CML 2001) de la Fachada Natural Aljibe.

\begin{tabular}{|c|c|c|c|c|c|c|c|c|}
\hline \multirow{2}{*}{ Categoría } & \multirow{2}{*}{ Indicador kg } & \multirow{2}{*}{ Total } & \multicolumn{2}{|c|}{ Capa vegetada } & \multicolumn{2}{c|}{ Estructura metálica } & \multicolumn{2}{c|}{ Capas de cerramiento } \\
\cline { 3 - 8 } & & $\mathbf{k g}$ & $\mathbf{\%}$ & $\mathbf{k g}$ & $\mathbf{\%}$ & $\mathbf{k g}$ & $\mathbf{\%}$ \\
\hline AA & Sb-eq. & $9,31 \mathrm{E}-01$ & $6,19 \mathrm{E}-01$ & 66,5 & $1,85 \mathrm{E}-01$ & 19,8 & $1,27 \mathrm{E}-01$ & 13,6 \\
\hline A & SO2-eq. & $5,71 \mathrm{E}-01$ & $3,77 \mathrm{E}-01$ & 66,1 & $1,05 \mathrm{E}-01$ & 18,5 & $8,83 \mathrm{E}-02$ & 15,5 \\
\hline Eu & Fosfa.-eq. & $1,10 \mathrm{E}-01$ & $8,39 \mathrm{E}-02$ & 76,6 & $1,34 \mathrm{E}-02$ & 12,2 & $1,23 \mathrm{E}-02$ & 11,2 \\
\hline CG & CO2-eq. & $1,09 \mathrm{E}+02$ & $6,52 \mathrm{E}+01$ & 60,1 & $2,31 \mathrm{E}+01$ & 20,9 & $2,09 \mathrm{E}+01$ & 18,9 \\
\hline AO & R11-eq. & $1,06 \mathrm{E}-05$ & $8,87 \mathrm{E}-06$ & 83,9 & $4,09 \mathrm{E}-07$ & 3,9 & $1,29 \mathrm{E}-06$ & 12,2 \\
\hline OF & C2H4-eq. & $6,08 \mathrm{E}-02$ & $3,40 \mathrm{E}-02$ & 55,9 & $1,76 \mathrm{E}-02$ & 28,9 & $9,26 \mathrm{E}-03$ & 15,2 \\
\hline TH & DCB-eq. & $6,89 \mathrm{E}+01$ & $1,05 \mathrm{E}+01$ & 15,2 & $5,35 \mathrm{E}+01$ & 77,7 & $4,86 \mathrm{E}+00$ & 7,1 \\
\hline EAD & DCB-eq. & $7,06 \mathrm{E}-01$ & $4,23 \mathrm{E}-01$ & 59,9 & $1,88 \mathrm{E}-01$ & 26,7 & $9,52 \mathrm{E}-02$ & 13,5 \\
\hline EAM & DCB-eq. & $2,22 \mathrm{E}+04$ & $1,13 \mathrm{E}+04$ & 50,8 & $8,35 \mathrm{E}+03$ & 37,6 & $2,57 \mathrm{E}+03$ & 11,6 \\
\hline
\end{tabular}

Tabla 7. Evaluación de las categorías de impacto Ecoindicador 99) de la Fachada Natural Aljibe.

\begin{tabular}{|c|c|c|c|c|c|c|c|c|}
\hline \multirow{2}{*}{ Categoría } & \multirow{2}{*}{ Indicador } & \multirow{2}{*}{ Total } & \multicolumn{2}{|c|}{ Capa vegetada } & \multicolumn{2}{|c|}{ Estructura metálica } & \multicolumn{2}{|c|}{ Capas de cerramiento } \\
\hline & & & Valor & $\%$ & Valor & $\%$ & Valor & $\%$ \\
\hline $\mathrm{A} / \mathrm{E}$ & $\mathrm{PDF} \cdot \mathrm{m}^{2} \cdot \mathrm{a}$ & $2,15 \mathrm{E}+\mathrm{OO}$ & $1,31 \mathrm{E}+\mathrm{Oo}$ & 60,9 & $4,46 \mathrm{E}-\mathrm{O} 1$ & 20,8 & $3,94 \mathrm{E}-\mathrm{O} 1$ & 18,4 \\
\hline $\mathrm{E}$ & $\mathrm{PDF} \cdot \mathrm{m}^{2} \cdot \mathrm{a}$ & $4,11 \mathrm{E}+\mathrm{OO}$ & $5,26 \mathrm{E}-\mathrm{O} 1$ & 12,8 & $3,21 \mathrm{E}+\mathrm{OO}$ & 78,0 & $3,76 \mathrm{E}-\mathrm{O} 1$ & 9,2 \\
\hline $\mathrm{C}$ & DALY & 4,11E-o6 & $1,98 \mathrm{E}-06$ & 48,2 & $1,62 \mathrm{E}-\mathrm{o} 6$ & 39,4 & 5,09E-07 & 12,4 \\
\hline $\mathrm{CC}$ & DALY & $6,84 \mathrm{E}-06$ & $-2,31 \mathrm{E}-06$ & $-34,0$ & 4,79E-06 & 70,0 & $4,36 \mathrm{E}-\mathrm{o} 6$ & 64,0 \\
\hline $\mathrm{CO}$ & DALY & $1,11 \mathrm{E}-\mathrm{o} 8$ & 9,34E-09 & 83,9 & $4,31 \mathrm{E}-10$ & 3,9 & $1,36 \mathrm{E}-09$ & 12,2 \\
\hline RI & DALY & $1,60 \mathrm{E}-\mathrm{O} 7$ & $1,06 \mathrm{E}-07$ & 65,8 & $2,79 \mathrm{E}-\mathrm{o} 8$ & 17,4 & $2,69 \mathrm{E}-08$ & 16,8 \\
\hline IR & DALY & 7,71E-O5 & $3,66 \mathrm{E}-\mathrm{O} 5$ & 47,4 & $2,71 \mathrm{E}-\mathrm{O} 5$ & 35,2 & $1,34 \mathrm{E}-\mathrm{O} 5$ & 17,4 \\
\hline OR & DALY & 6,01E-o8 & $3,72 \mathrm{E}-\mathrm{o} 8$ & 61,9 & $1,03 \mathrm{E}-\mathrm{o} 8$ & 17,2 & $1,25 \mathrm{E}-\mathrm{o} 8$ & 20,9 \\
\hline $\mathrm{CF}$ & MJ & $1,8 \mathrm{oE}+02$ & $1,41 \mathrm{E}+\mathrm{O} 2$ & 78,4 & $1,54 \mathrm{E}+\mathrm{O} 1$ & 8,6 & $2,34 \mathrm{E}+01$ & 13,0 \\
\hline $\mathrm{M}$ & MJ & $6,44 \mathrm{E}-01$ & $1,89 \mathrm{E}-\mathrm{O} 2$ & 2,9 & $6,11 \mathrm{E}-\mathrm{O} 1$ & 94,9 & $1,41 \mathrm{E}-\mathrm{O} 2$ & 2,2 \\
\hline
\end{tabular}


CML 2001

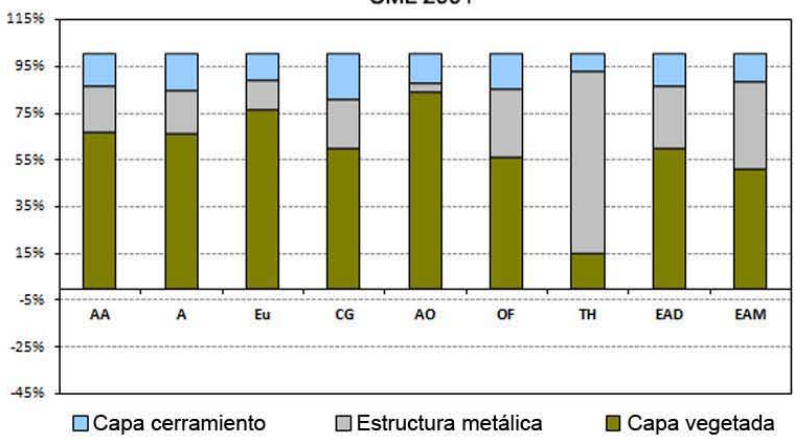

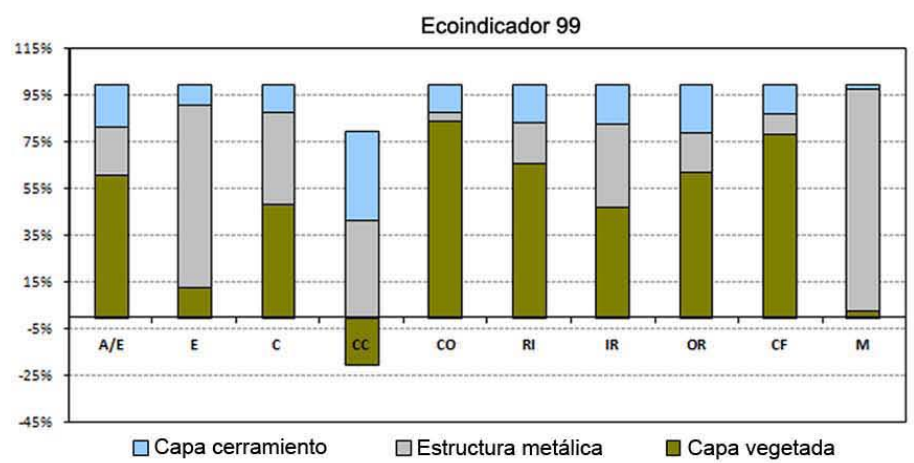

Figura 2. Contribución relativa a las categorías de impacto CML 2001 y Ecoindicador 99) de los sistemas que componen la Fachada Natural Aljibe.

Tabla 8. Evaluación de las categorías de impacto (CML 2001) asociadas al transporte de la Fachada Natural Aljibe hasta la obra.

\begin{tabular}{|c|c|c|c|c|c|c|c|c|}
\hline \multirow{2}{*}{ Categoría } & \multirow{2}{*}{ Indicador kg } & \multirow{2}{*}{ Total } & \multicolumn{2}{|c|}{ Capa vegetada } & \multicolumn{2}{|c|}{ Estructura metálica } & \multicolumn{2}{|c|}{ Capas de cerramiento } \\
\hline & & & kg & $\%$ & kg & $\%$ & kg & $\%$ \\
\hline AA & Sb-eq. & 9,6oE-03 & $8,47 \mathrm{E}-03$ & 88,2 & 2,06E-04 & 2,2 & $9,25 \mathrm{E}-04$ & 9,6 \\
\hline $\mathrm{A}$ & SO2-eq. & $8,17 \mathrm{E}-03$ & $7,13 \mathrm{E}-\mathrm{O} 3$ & 87,4 & 1,88E-04 & 2,3 & $8,44 \mathrm{E}-\mathrm{O} 4$ & 10,3 \\
\hline $\mathrm{Eu}$ & Fosfa.-eq. & 1,40E-03 & 1,22E-O3 & 87,3 & $3,25 \mathrm{E}-\mathrm{O} 5$ & 2,3 & $1,46 \mathrm{E}-04$ & 10,4 \\
\hline CG & CO2-eq. & $1,36 \mathrm{E}+00$ & $1,19 \mathrm{E}+\mathrm{Oo}$ & 87,7 & $3,05 \mathrm{E}-02$ & 2,2 & $1,37 \mathrm{E}-\mathrm{O} 1$ & 10,1 \\
\hline $\mathrm{AO}$ & R11-eq. & 2,70E-09 & 2,38E-09 & 88,2 & $5,81 \mathrm{E}-11$ & 2,2 & $2,60 \mathrm{E}-10$ & 9,6 \\
\hline OF & $\mathrm{C}_{2} \mathrm{H}_{4}$-eq. & $6,50 \mathrm{OE}-\mathrm{O} 4$ & $5,66 \mathrm{E}-04$ & 87,1 & $1,53 \mathrm{E}-05$ & 2,4 & $6,85 \mathrm{E}-\mathrm{O} 5$ & 10,5 \\
\hline $\mathrm{TH}$ & DCB-eq. & $4,27 \mathrm{E}-\mathrm{O} 2$ & $3,73 \mathrm{E}-02$ & 87,3 & 9,93E-04 & 2,3 & $4,45 \mathrm{E}-\mathrm{O} 3$ & 10,4 \\
\hline EAD & DCB-eq. & 1,62E-03 & $1,43 \mathrm{E}-\mathrm{O} 3$ & 88,2 & $3,49 \mathrm{E}-05$ & 2,2 & $1,56 \mathrm{E}-04$ & 9,7 \\
\hline EAM & DCB-eq. & $2,52 \mathrm{E}+\mathrm{O} 1$ & $2,22 \mathrm{E}+\mathrm{O} 1$ & 88,2 & $5,41 \mathrm{E}-\mathrm{O} 1$ & 2,2 & $2,42 \mathrm{E}+\mathrm{Oo}$ & 9,6 \\
\hline
\end{tabular}

Tabla 9. Evaluación de las categorías de impacto (Ecoindicador 99) asociadas al transporte de la Fachada Natural Aljibe.

\begin{tabular}{|c|c|c|c|c|c|c|c|c|}
\hline \multirow{2}{*}{ Categoría } & \multirow{2}{*}{ Indicador } & \multirow{2}{*}{ Total } & \multicolumn{2}{|c|}{ Capa vegetada } & \multicolumn{2}{|c|}{ Estructura metálica } & \multicolumn{2}{|c|}{ Capas de cerramiento } \\
\hline & & & Valor & $\%$ & Valor & $\%$ & Valor & $\%$ \\
\hline $\mathrm{A} / \mathrm{E}$ & $\mathrm{PDF} \cdot \mathrm{m}^{2} \cdot \mathrm{a}$ & $6,14 \mathrm{E}-\mathrm{O} 2$ & $5,36 \mathrm{E}-\mathrm{O} 2$ & 87,3 & $1,42 \mathrm{E}-\mathrm{O} 3$ & 2,3 & $6,38 \mathrm{E}-\mathrm{O} 3$ & 10,4 \\
\hline $\mathrm{E}$ & $\mathrm{PDF} \cdot \mathrm{m}^{2} \cdot \mathrm{a}$ & 1,10E-03 & 9,68E-04 & 88,2 & $2,36 \mathrm{E}-\mathrm{O} 5$ & 2,2 & $1,06 \mathrm{E}-04$ & 9,6 \\
\hline $\mathrm{C}$ & DALY & $1,91 \mathrm{E}-08$ & $1,68 \mathrm{E}-08$ & 88,2 & $4,10 \mathrm{E}-10$ & 2,2 & $1,84 \mathrm{E}-09$ & 9,6 \\
\hline $\mathrm{CC}$ & DALY & $2,85 \mathrm{E}-\mathrm{O} 7$ & $2,5 \mathrm{OE}-\mathrm{O} 7$ & 87,7 & 6,39E-09 & 2,2 & $2,86 \mathrm{E}-\mathrm{o} 8$ & 10,1 \\
\hline $\mathrm{CO}$ & DALY & $2,84 \mathrm{E}-12$ & $2,51 \mathrm{E}-12$ & 88,2 & $6,11 \mathrm{E}-14$ & 2,2 & $2,74 \mathrm{E}-13$ & 9,6 \\
\hline RI & DALY & $7,08 \mathrm{E}-11$ & $6,25 \mathrm{E}-11$ & 88,2 & $1,52 \mathrm{E}-12$ & 2,2 & $6,82 \mathrm{E}-12$ & 9,6 \\
\hline IR & DALY & $1,15 \mathrm{E}-06$ & 1,0oE-06 & 87,2 & $2,69 \mathrm{E}-08$ & 2,3 & $1,20 \mathrm{E}-07$ & 10,5 \\
\hline OR & DALY & $6,23 \mathrm{E}-10$ & $5,41 \mathrm{E}-10$ & 86,9 & $1,49 \mathrm{E}-11$ & 2,4 & $6,66 \mathrm{E}-11$ & 10,7 \\
\hline $\mathrm{CF}$ & MJ & $2,26 \mathrm{E}+\mathrm{Oo}$ & $2, \mathrm{ooE}+\mathrm{OO}$ & 88,2 & $4,87 \mathrm{E}-02$ & 2,2 & $2,18 \mathrm{E}-\mathrm{O} 1$ & 9,6 \\
\hline M & MJ & $6,14 \mathrm{E}-\mathrm{O} 5$ & $5,42 \mathrm{E}-\mathrm{O} 5$ & 88,2 & $1,32 \mathrm{E}-06$ & 2,2 & $5,92 \mathrm{E}-\mathrm{o} 6$ & 9,6 \\
\hline
\end{tabular}

de materiales con igual comportamiento en el vertedero. En cada grupo se incluye la etapa de transporte hasta vertedero y su tratamiento en él. Asimismo, se incluyen en ambas metodologías la contribución relativa al impacto total de cada uno de los grupos de materiales según su comportamiento en el vertedero. Se comprueba que una de las mayores contribuciones a los impactos, durante esta etapa del ciclo de vida, la constituyen los restos orgánicos procedentes del sustrato y todos los materiales plásticos de la capa vegetada. Otra contribución importante es la debida a los materiales inertes constituidos por los paneles de yeso y paneles de cemento. La contribución a los impactos por parte de la etapa de transporte hasta vertedero no es superior al $7 \%$, frente a los impactos del proceso de fin de vida para cada uno de los grupos de materiales.

\subsection{Resultados totales}

En la Figura 3 se muestra, a modo de resumen, las categorías de impacto según las dos metodologías empleadas y para cada una de las etapas analizadas: suministro de materias primas, transporte y fabricación, transporte hasta la obra y fin de vida.

\subsection{Información de utilidad para declaraciones ambientales de producto}

De los resultados generados por el análisis de los impactos de ciclo de vida de la Fachada Natural Aljibe, se extrae información relevante para la elaboración de una DAP con límites del sistema «de la cuna a la puerta con opciones» (apartado 5.2 
Tabla 10. Evaluación de las categorías de impacto (CML 2001) asociadas a los procesos de fin de vida de la Fachada Natural Aljibe.

\begin{tabular}{|c|c|c|c|c|c|c|c|c|c|c|c|c|}
\hline \multirow{2}{*}{ Categoría } & \multirow{2}{*}{$\begin{array}{c}\text { Indicador } \\
\mathbf{k g}\end{array}$} & \multirow{2}{*}{ Total } & \multicolumn{2}{|c|}{ Acero } & \multicolumn{2}{|c|}{ Basura orgánica } & \multicolumn{2}{|c|}{ Lana mineral } & \multicolumn{2}{|c|}{ Material inerte } & \multicolumn{2}{|c|}{$\begin{array}{c}\text { Mezcla } \\
\text { de plásticos }\end{array}$} \\
\hline & & & kg & $\%$ & kg & $\%$ & kg & $\%$ & kg & $\%$ & kg & $\%$ \\
\hline $\mathrm{AA}$ & Sb-eq. & $7,23 \mathrm{E}-\mathrm{O} 3$ & $2,22 \mathrm{E}-\mathrm{o6}$ & 0,03 & $2,84 \mathrm{E}-\mathrm{O} 3$ & 39,19 & 3,08E-04 & 4,26 & 2,82E-03 & 39,04 & $1,26 \mathrm{E}-03$ & 17,48 \\
\hline $\mathrm{A}$ & SO2-eq. & $7,63 \mathrm{E}-03$ & $2,48 \mathrm{E}-\mathrm{o} 6$ & 0,03 & $3,37 \mathrm{E}-03$ & 44,21 & $1,90 \mathrm{E}-\mathrm{O} 4$ & 2,49 & $3,16 \mathrm{E}-03$ & 41,42 & $9,04 \mathrm{E}-04$ & 11,86 \\
\hline $\mathrm{Eu}$ & & & & & & & & & & & $1,34 \mathrm{E}-01$ & 77,77 \\
\hline CG & & $1,67 \mathrm{E}+01$ & $3,23 \mathrm{E}-\mathrm{O} 4$ & 0,00 & $1,51 \mathrm{E}+$ & 90,23 & $2,54 \mathrm{E}-\mathrm{O} 2$ & 0,15 & & 4,59 & $8,39 \mathrm{E}-01$ & 5,03 \\
\hline $\mathrm{AO}$ & & & $4,97 \mathrm{E}-12$ & & & & & 13,81 & & & $2,85 \mathrm{E}-\mathrm{o} 8$ & 52,01 \\
\hline OF & $\mathrm{C}_{2} \mathrm{H}_{4}$-eq. & 4,98E-03 & $3,15 \mathrm{E}-07$ & 0,01 & 4,18E-O3 & 83,91 & $3,10 \mathrm{E}-\mathrm{O} 5$ & 0,62 & 5,01E-04 & 10,07 & $2,68 \mathrm{E}-04$ & 5,38 \\
\hline TH & & & & 0,02 & $1,96 \mathrm{E}-02$ & 26,70 & 2,32E-O3 & 3,16 & $1,83 \mathrm{E}-02$ & 24,87 & $3,33 \mathrm{E}-02$ & 45,25 \\
\hline EAD & DCB-eq. & 1,98E-02 & 4,43 E-07 & 0,00 & $1,55^{\mathrm{E}-03}$ & 7,82 & $1,85 \mathrm{E}-04$ & 0,93 & $1,94 \mathrm{E}-03$ & 9,80 & $1,61 \mathrm{E}-02$ & 81,45 \\
\hline EAM & DCB-eq. & $2,20 \mathrm{E}+\mathrm{O} 2$ & $7,20 \mathrm{E}-02$ & 0,03 & $7,95 \mathrm{E}+01$ & 36,10 & $1,95 \mathrm{E}+00$ & 0,88 & $9,19 \mathrm{E}+01$ & 41,74 & $4,68 \mathrm{E}+01$ & 21,24 \\
\hline
\end{tabular}

Tabla 11. Evaluación de las categorías de impacto (Ecoindicador 99) asociadas a los procesos de fin de vida de la Fachada Natural Aljibe.

\begin{tabular}{|c|c|c|c|c|c|c|c|c|c|c|c|c|}
\hline \multirow[t]{2}{*}{ Categoría } & \multirow[t]{2}{*}{ Indicador } & \multirow[t]{2}{*}{ Total } & \multicolumn{2}{|c|}{ Acero } & \multicolumn{2}{|c|}{$\begin{array}{c}\text { Basura } \\
\text { orgánica }\end{array}$} & \multicolumn{2}{|c|}{ Lana mineral } & \multicolumn{2}{|c|}{ Material inerte } & \multicolumn{2}{|c|}{$\begin{array}{c}\text { Mezcla } \\
\text { de plásticos }\end{array}$} \\
\hline & & & Valor & $\%$ & Valor & $\%$ & Valor & $\%$ & Valor & $\%$ & Valor & $\%$ \\
\hline $\mathrm{A} / \mathrm{E}$ & $\mathrm{PDF} \cdot \mathrm{m}^{2} \cdot \mathrm{a}$ & $4,25 \mathrm{E}-\mathrm{O} 2$ & $1,33 \mathrm{E}-05$ & 0,03 & $1,86 \mathrm{E}-02$ & 43,78 & 1,30E-O3 & 3,07 & $1,69 \mathrm{E}-\mathrm{O} 2$ & 39,77 & $5,68 \mathrm{E}-03$ & 13,35 \\
\hline $\mathrm{E}$ & $\mathrm{PDF} \cdot \mathrm{m}^{2} \cdot \mathrm{a}$ & $2,52 \mathrm{E}-\mathrm{O} 2$ & $2,87 \mathrm{E}-07$ & 0,00 & $1,89 \mathrm{E}-02$ & 74,92 & $3,46 \mathrm{E}-\mathrm{O} 4$ & 1,38 & $3,34 \mathrm{E}-03$ & 13,25 & $2,63 \mathrm{E}-03$ & 10,45 \\
\hline $\mathrm{C}$ & DALY & $5,80 \mathrm{E}-\mathrm{O} 7$ & $7,21 \mathrm{E}-12$ & 0,00 & $5,17 \mathrm{E}-07$ & 89,10 & $6,89 \mathrm{E}-10$ & 0,12 & $1,11 \mathrm{E}-\mathrm{o} 8$ & 1,91 & $5,15 \mathrm{E}-\mathrm{o} 8$ & 8,87 \\
\hline $\mathrm{CC}$ & ALY & 3,06E-06 & $6,75^{\mathrm{E}-11}$ & 0,00 & $2,74 \mathrm{E}-06$ & 89,81 & $5,28 \mathrm{E}-09$ & 0,17 & $1,49 \mathrm{E}-07$ & 4,89 & $1,57 \mathrm{E}-07$ & 5,13 \\
\hline $\mathrm{CO}$ & & & & & & & $7,94 \mathrm{E}-12$ & 13,80 & $68 \mathrm{E}-12$ & 11,61 & $2,99 \mathrm{E}-11$ & 51,97 \\
\hline RI & ALY & $6,99 \mathrm{E}-10$ & $1,32 \mathrm{E}-13$ & 0,02 & $3,25 \mathrm{E}-10$ & 46,43 & $1,73 \mathrm{E}-11$ & 2,47 & $1,68 \mathrm{E}-10$ & 24,09 & $1,89 \mathrm{E}-10$ & 26,99 \\
\hline IR & & & $9,16 \mathrm{E}-10$ & 0,05 & & 33,12 & $3,26 \mathrm{E}-08$ & 1,62 & $1,17 \mathrm{E}-06$ & 57,93 & $1,47 \mathrm{E}-07$ & 7,28 \\
\hline OR & DALY & $8,58 \mathrm{E}-09$ & $4,27 \mathrm{E}-13$ & 0,00 & $7,29 \mathrm{E}-09$ & 85,02 & $5,27 \mathrm{E}-11$ & 0,61 & $7,25 \mathrm{E}-10$ & 8,45 & $5,07 \mathrm{E}-10$ & 5,91 \\
\hline $\mathrm{CF}$ & & $1,69 \mathrm{E}+00$ & 5,08E-04 & 0,03 & $6,84 \mathrm{E}-\mathrm{O} 1$ & 40,47 & $7,12 \mathrm{E}-\mathrm{O} 2$ & 4,21 & $6,47 \mathrm{E}-\mathrm{O} 1$ & 38,26 & $2,88 \mathrm{E}-\mathrm{O} 1$ & 17,04 \\
\hline $\mathrm{M}$ & MJ & $2,53 \mathrm{E}-\mathrm{O} 4$ & $8,19 \mathrm{E}-09$ & 0,00 & $2,12 \mathrm{E}-\mathrm{O} 5$ & 8,37 & 3,61E-05 & 14,24 & $1,04 \mathrm{E}-05$ & 4,12 & $1,86 \mathrm{E}-04$ & 73,28 \\
\hline
\end{tabular}
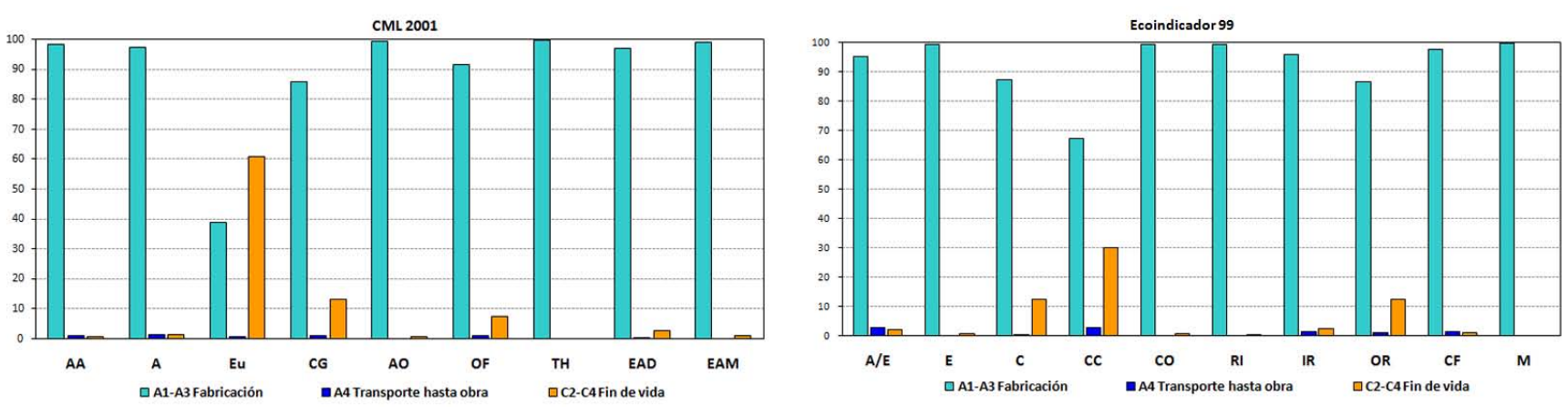

Figura 3. Contribución relativa de las etapas analizadas en el ciclo de vida a las categorías de impacto totales (CML 2001 y Ecoindicador 99) de la Fachada Natural Aljibe.

de la norma UNE-EN 15804). Se utilizará el concepto de unidad declarada, en lugar de unidad funcional, por no conocerse los escenarios de uso de la fachada a nivel edificio (apartado 6.3.2 de la norma UNE-EN 15804). La unidad declarada es «1 $\mathrm{m}^{2}$ de cerramiento vertical opaco». Asimismo, se indica que los límites del sistema declarados incluyen las siguientes etapas del ciclo de vida (según definiciones que aparecen en la norma UNE-EN 15804):

A1. Suministro de materias primas,

A2. Transporte,

A3. Fabricación,

A4. Transporte (hasta obra),

C2. Transporte (hasta tratamiento de fin de vida),

C4. Vertido.

Las categorías de impacto evaluadas corresponden a la metodología CML 2001, pero únicamente se evalúan las citadas en la norma UNE-EN 15804:

\section{CONCLUSIONES}

Tras el estudio realizado sobre los impactos ambientales asociados a las fases de fabricación, construcción, mantenimiento, deconstrucción y fin de vida de la nueva solución de fachada vegetal propuesta, se llega a las siguientes conclusiones:

- La contribución más importante a las categorías de impacto del ciclo de vida corresponde a la etapa de fabricación de los componentes de la fachada. La mayor contribución a los impactos en el suministro de materias primas y materiales auxiliares, en el transporte de éstos hasta el lugar de fabricación y en la fabricación de la Fachada Natural Aljibe se debe a la capa vegetada formada por el contenedor NATURPANEL® Aljibe, el acabado superficial y los elementos auxiliares. La segunda mayor contribución se debe a la estructura metálica, siendo por tanto la menor contribución la de las capas de cerramiento. 
Tabla 12. Evaluación de impactos (CML 2001) asociados a las etapas A1-A3, A4 y C2-C4.

\begin{tabular}{|c|c|c|c|c|}
\hline $\begin{array}{l}\text { Parámetro } \\
\text { evaluado }\end{array}$ & Indicador $\mathrm{kg}$ & A1-A3 & A4 & $\mathrm{C}_{2}-\mathrm{C}_{4}$ \\
\hline $\mathrm{AA}$ & Sb-eq. & $6,21 \mathrm{E}-\mathrm{O} 1$ & $5,37 \mathrm{E}-\mathrm{O} 3$ & $5,18 \mathrm{E}-\mathrm{O} 3$ \\
\hline $\mathrm{A}$ & SO2-eq. & $3,82 \mathrm{E}-\mathrm{O} 1$ & $4,60 \mathrm{E}-\mathrm{O} 3$ & $5,49 \mathrm{E}-\mathrm{O} 3$ \\
\hline $\mathrm{Eu}$ & Fosfa.-eq. & $6,77 \mathrm{E}-01$ & $7,90 \mathrm{E}-04$ & $8,64 \mathrm{E}-\mathrm{O} 2$ \\
\hline CG & CO2-eq. & $2,96 \mathrm{E}+01$ & $7,64 \mathrm{E}-01$ & $8,74 \mathrm{E}+\mathrm{Oo}$ \\
\hline $\mathrm{AO}$ & R11-eq. & 6,14E-06 & 1,51E-09 & $3,43 \mathrm{E}-08$ \\
\hline OF & $\mathrm{C}_{2} \mathrm{H}_{4}$-eq. & $4,38 \mathrm{E}-02$ & $3,67 \mathrm{E}-04$ & $2,75 \mathrm{E}-03$ \\
\hline
\end{tabular}

Dentro de la capa vegetada, la mayor contribución se debe al acabado superficial, compuesto por el abono, la fibra de coco, el poliestireno expandido y los sacos de fieltro. De todos estos elementos, los que mayor peso tienen sobre las categorías de impacto son la fibra de coco, el poliestireno expandido y el fieltro. La segunda mayor contribución a

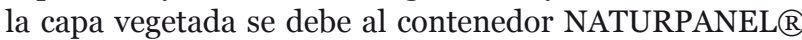
Aljibe, en el que los mayores impactos se atribuyen al proceso de inyección.

- La etapa de transporte hasta el lugar de construcción es la que presenta menor contribución a todo el ciclo de vida analizado, pudiéndose considerar despreciable en todas las categorías de impacto analizadas.

- Para la etapa de fin de vida (transporte y proceso de fin de vida) presenta, para la mayoría de las categorías de impacto analizadas, una contribución muy reducida al impacto de todo el ciclo de vida. Para esta etapa, las únicas categorías de impacto que sí contribuyen significativamente con respecto a las etapas de suministro de materias primas y materiales auxiliares, transporte de éstos hasta el lugar de fabricación y fabricación son la eutrofización, calentamiento global y formación de oxidantes fotoquímicos, para la metodología CML 2001. En concreto, en el caso de la eutrofización, los impactos de la etapa de fin de vida superan a los impactos de la etapa de fabricación. Para las categorías de impacto asociadas a la metodología Ecoindicador 99 se destaca una mayor contribución de carcinógenos, cambio climático y orgánicos respirables.
Con el desarrollo de este análisis se ha obtenido información útil de los aspectos y componentes más problemáticos en el desempeño ambiental de la fachada evaluada, abriendo así el camino hacia posibles acciones de reducción de impactos por parte del fabricante.

\section{AGRADECIMIENTOS}

Este trabajo de investigación ha sido realizado gracias a los fondos públicos destinados a la investigación científica, desarrollo e innovación tecnológica con el que cuenta el sistema español de Ciencia, Tecnología y Empresa. El grupo de investigación ABIO-UPM, perteneciente a la Universidad Politécnica de Madrid, agradece a la empresa Intemper S.L. la confianza depositada en nosotros para llevar a cabo este estudio, siendo tan solo una pequeña parte de todo el proyecto «SOS Natura, Soluciones Arquitectónicas Vegetales» perteneciente al programa INNPACTO del Ministerio de Ciencia e Innovación y formado por AmetsLab, BambHaus y Cidemco.

Asimismo, los autores agradecen el aporte técnico recibido para la realización de esta investigación a Luis A. Alonso, Mariana Chanampa, Jesús García, Ma Carolina Hernández, Francesca Olivieri, Jorge Orondo, Regina Pastor, Ma del Alba Vicente y Pilar Vidal, pertenecientes a los equipos de simulación, monitorización y construcción del grupo de investigación ABIO-UPM.

\section{REFERENCIAS}

(1) International Energy Agency. (2011). World Energy Outlook 2011. http://www.iea.org/publications/freepublications/ publication/es_spanish.pdf.

(2) Rivela, B., Cuerda, I., Olivieri, F., Bedoya, C., Neila, J. (2012). Análisis de Ciclo de Vida para el ecodiseño del sistema Intemper TF de cubierta ecológica aljibe. Materiales de la construcción, 63(309): 131-145, doi: http://dx.doi.org/10.3989/ mc.2012.02611.

(3) Fullana, P., Puig, R. (1997). Análisis del Ciclo de Vida. p.143, Barcelona: Rubes.

(4) Benveniste, G., Gazulla, C., Fullana, P., Celades, I., Ros, T., Zaera, V., Godes, B. (2011). Análisis de ciclo de vida y reglas de categoría de producto en la construcción. El caso de las baldosas cerámicas. Informes de la construcción, 63(522): 71-81, doi: http://dx.doi.org/10.3989/ic.10.034.

(5) AENOR. (2006). UNE-EN ISO 14040:2006 - Gestión ambiental. Análisis de ciclo de vida. Principios y marco de referencia. Asociación Española de Normalización (AENOR).

(6) AENOR. (2006). UNE-EN ISO 14044:2006 - Gestión ambiental. Análisis de ciclo de vida. Requisitos y directrices. Asociación Española de Normalización (AENOR).

(7) AENOR. (2012). UNE-EN 15804:2012 - Sostenibilidad en la construcción. Declaraciones ambientales de producto. Reglas de categorías de productos básicas para productos de construcción. Asociación Española de Normalización (AENOR).

(8) Rebitzer, G., Fullana, P., Weidema, B.P., Jolliet, O. (2003). Recycling, Close-Loop Economy, Secondary Resources. The international journal of life cycle assessment, 8(2): 106-108, doi: http://dx.doi.org/10.1007/BFo2978434.

(9) Fullana-i-Palmer, P., Puig, R., Bala, A., Baquero, G., Riba, J., Raugei, M. (2011). From Life Cycle Assessment to Life Cycle Management: A Case Study On Industrial Waste Management Policy Making. Journal of industrial ecology, 15(3): 458475, doi: http://dx.doi.org/10.1111/j.1530-9290.2011.00338.x. 
(10) Ministerio de Fomento - Gobierno de España. (2006). Código Técnico de la Edificación CTE. http://www.fomento.gob.es.

(11) WWF.(2011). Observatorio de la Electricidad. http://www.wwf.es/que_hacemos/cambio_climatico/nuestras_soluciones/energias_renovables/observatorio_de_la_electricidad/

(12) European Commission. (2006). Directive 2006/38/EC of the European Parliament and of the Council of 17 May 2006 amending Directive 1999/62/EC on the charging of heavy goods vehicles for the use of certain infrastructures. http://eurlex.europa.eu/LexUriServ/LexUriServ.do?uri=OJ:L:2006:157:0008:0023:EN:PDF.

(13) Goedkoop, M., Spriensma, R. (2000). The eco-indicator 99. A damage oriented method for life cycle impact assessment. p.142, Amersfoort, the Nederthlands: Pré Consultants B.V.

(14) Guinée, J. (2002). Handbook on Life Cycle Assessment. Operational Guide to the ISO Standards. p.708, Dordrecht, the Nederthlands: Kluwer Academic Publishers. 